\title{
Fluorescein angiography in anterior segment ischaemia
}

\author{
D. L. EASTY AND A. H. CHIGNELL \\ Moorfields Eye Hospital, City Road, London
}

The clinical signs which result from anterior segment necrosis have been described and
documented on many occasions, the characteristic features which affect the iris being a fixed oval pupil, sectoral atrophy of the stroma with tortuosity of the stromal fibres, and 응 atrophy of sectors of the sphincter (Wilson and Irvine, I955; Boniuk and Zimmerman, iv I 96 I ; Knox, I965; Crock, I967; Sanders and Hoyt, I969; O’Day, Hoyt, and Crock, I970).. It is upon these signs that the diagnosis often relies. In the early stages of an ischaemic $\vec{\infty}$ attack, particularly after surgical procedures, the diagnosis may be obscured by corneal $\stackrel{\circ}{\supset}$ oedema and infiltration, and by an acute fibrinous reaction in the anterior chamber. The signs in the anterior segment may be remarkably similar to those produced by other stimuli such as operative trauma or infection, and may lead to misdiagnosis and erroneous therapy.

Fluorescein angiography has recently been introduced as a technique for the study of the $\frac{\vec{\omega}}{\omega}$ iris vessels in various diseases (Mitsui, Matsubara, and Kanagawa, I969; Déodati, Bec, Labro, and Camezind, 1969; Bron and Easty, 1970). Vannas (1969) described in some detail his technique of photography and demonstrated the angiographic findings in pseudoexfoliation of the lens capsule and in various types of glaucoma. The effects of iris disease on the fluorescein pattern have been reported by other workers; Cobb (1968) noted the fluorescence of vascular tufts at the pupil margin, which he ater noted were particularly common in myotonic dystrophy (Cobb, Shilling, and Chisholm, 1970). Other studies have been made into the vasculature of the iris in diabetes mellitus (Jensen and Lundbaek, I968; Baggesen, I969) and iris tumours (Cheng, Bron, and Easty, I97 I).

Most of these reports have emphasized the significance of vascular damage presenting as fluorescein leakage into the iris stroma or anterior chamber. However, there have been few reports concerning its value in determining the efficiency of the perfusion of the iris vessels with blood in ischaemic conditions (Chignell and Easty, I97 I).

A study was therefore designed to examine, by means of fluorescein angiography, the 윽 vascular anatomy in patients with recognized ischaemic conditions of the iris. Thereafter, $\frac{D}{0}$ having assessed the findings in established cases of ischaemia, it was planned to investigate its diagnostic potential in suspected ischaemic states, particularly those following surgical $\mathbb{N}$ procedures such as retinal detachment operations. This paper reports the results of these $\tilde{\mathcal{D}}$ studies.

\section{Material and methods}

Fluorescein angiograms were performed in a total of 52 patients; 44 of them had undergone retinal detachment procedures and fifteen of these had suffered a definite attack of anterior segment $\stackrel{\mathcal{E}}{?}$ necrosis. Six patients with angle-closure glaucoma and two who had had ischaemia after strabismus surgery were also studied.

Detailed examination was performed with the slit lamp and the clinical signs were fully documented and photographed. Damage to the pigment epithelium of the iris was detected by transpupillary transillumination, and atrophy of the sphincter pupillae was assessed by carefully noting the pupillary 
response to light in all sectors. Stromal damage was observed with oblique illumination in the standard way.

TECHNIQUE OF FLUORESCEIN ANGIOGRAPHY

The Zeiss photo-slit lamp was modified in the following ways in order to make it suitable for fluorescein angiography of the iris. The power pack of the photo-slit lamp was replaced by a modified Zeiss Fundus camera power pack. With this arrangement and the number 4 flash setting, exposures at $2 \cdot 0$ to $2.5 \mathrm{sec}$. intervals could be achieved. The excitation filter used was of the interference type (Balzar: B4) and the barrier filter was Kodak Wratten I5. Photographs were taken at $\times$ I magnification and $5 \mathrm{ml}$. of 20 per cent. fluorescein solution were injected into the antecubital vein in all cases. Kodak Tri X Film was used (ASA 400) and this was developed for $12 \mathrm{~min}$. in ID i a $68^{\circ} \mathrm{F}$.

\section{Results}

\section{Fluorescein angiography in recognised ischaemia}

In the following paragraphs, selected examples of the fluorescein angiograms from patients who had suffered attacks of severe ischaemia are presented.

Severe ischaemia of the anterior segment may cause corneal oedema due to endothelial decompensation, and large dichotomously branching blood vessels may eventually enter the deep corneal stroma (Sanders and Hoyt, 1969).

An ischaemic attack resulted from a retinal detachment procedure in a 52 -year-old female during which the left eye was encircled with a silicone strap, cryothermy having been applied and subretinal fluid having been released. 6 months later she had developed a mature cataract which was treated by extracapsular extraction, followed by a capsulotomy at a later date. Fig. I $a$ demonstrates the clinical appearance 6 months after surgery. There is a gross degree of corneal stromal oedema and deep vessels can be seen invading its substance. Fig. I $b$ shows the fluorescein findings. The iris demonstrates gross leakage of fluorescein at an early stage, and the extent of the deep corneal vessels is well shown.

(a)

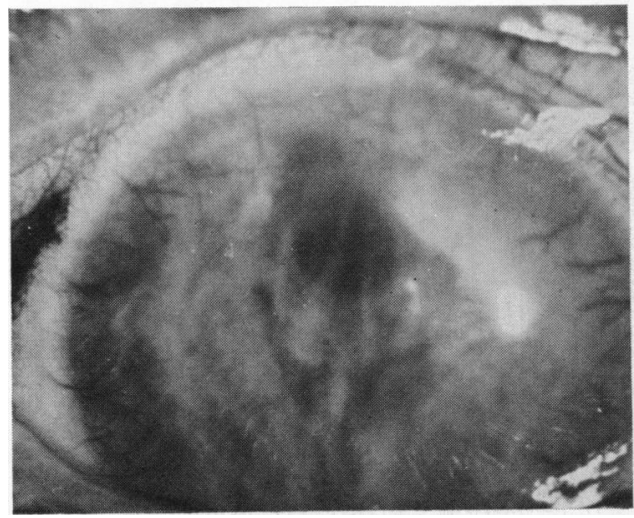

(b)

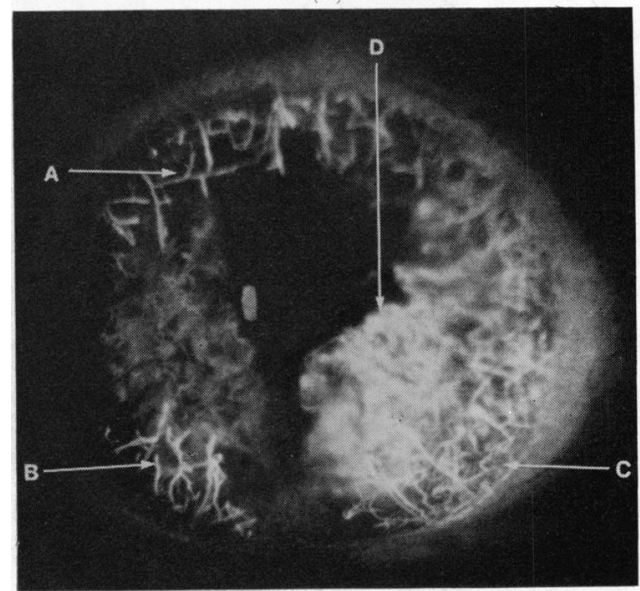

FIG. I a Gross anterior segment necrosis following detachment and cataract surgery

FIG. I $b$ Angiogram showing deep stromal vessels $(A, B, C)$ with heavy leakage from the iris $(D)$

The appearance of the iris in a 63 -year-old male patient during an attack of angle-closure glaucoma is shown in Fig. 2a. At the time of the examination the intraocular pressure was $40 \mathrm{~mm}$. $\mathrm{Hg}$. There is severe ischaemia of the iris, with early and dense leakage from vessels in a sector in the lower temporal quadrant. At a later stage (Fig. $2 b$ ) other vessels fluoresce and demonstrate an intense leakage into the stroma of the iris and aqueous humour. 


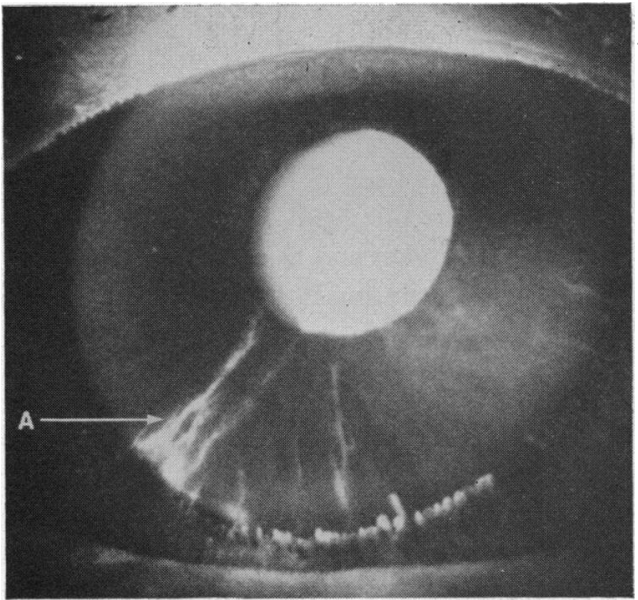

FI G. $2 a$ Angiogram in a patient with angle-closure glaucoma, showing early leakage $(A)$ and diffuse ischaemia (30 sec.)

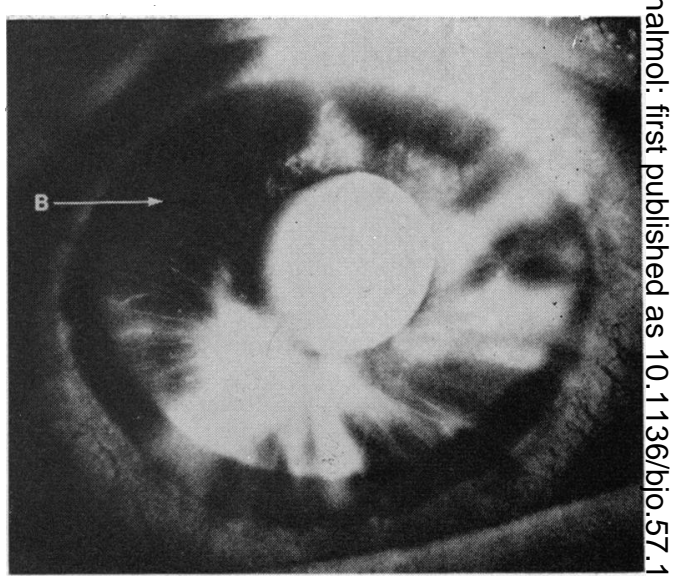

FIG. $2 b$ Late angiogram (I min. 54 sec.) demon- $\vec{\infty}$ strating heavy leakage produced by severe vascular $ᄋ$ damage with pronounced ischaemia in one sector $(B)-$

The appearance of the iris in a 52 -year-old male with a history of intermittent blurring of the vision in the right eye due to angle-closure glaucoma is shown in Fig. 3. The iris vessels demonstrate a distortion in their anatomy that correlates well with the clinical appearance of the iris after an $\overrightarrow{0}$ attack of acute glaucoma. Although reduced perfusion is apparent on the nasal side, it is noteworthy that there is not complete avascularity.

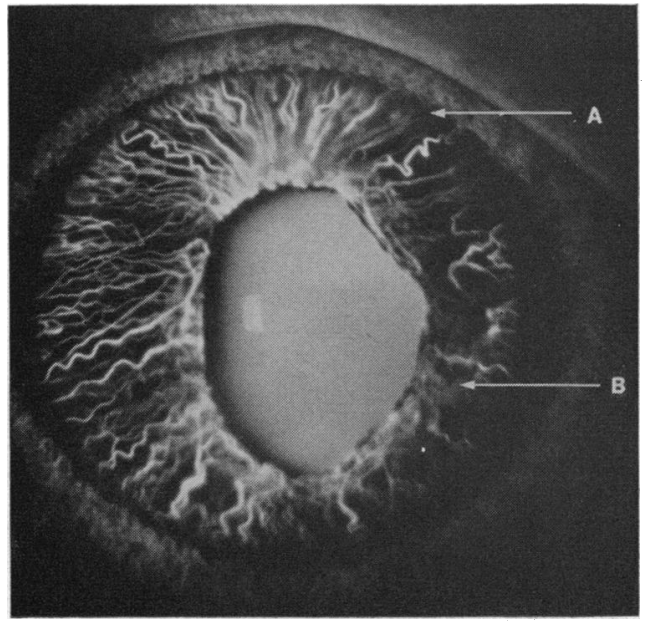

FIG. 3 Angiogram in angle-closure glaucoma at normal intraocular pressure. $A$ and $B$ demonstrate $\frac{O}{-}$. a sector of ischaemia of moderate severity

A 35-year-old male suffered an attack of anterior segment necrosis after cryotherapy, drainage of subretinal fluid, and encerclage with a $3-\mathrm{mm}$. silicone strap for a retinal detachment in the right eye. N Fig. $4 a$ (opposite) shows the appearance of the eye 6 months postoperatively, the stroma showing signs of atrophy in two places. Fig. $4 b$ shows the appearance of the iris on transillumination, and indicateso that the pigmentary epithelium is also deficient. The iris angiogram (Fig. $4 c$ ) demonstrates that the ischaemic area coincides with the clinically atrophic area while, in the opposite half of the iris, the perfusion of the vessels appears unaffected.

Certain patients with minimal signs in the iris after retinal detachment surgery were examined, in many of whom an ischaemic aetiology had not been evoked in the immediate $\stackrel{\mathbb{D}}{\Omega}$ postoperative period. 
(a)

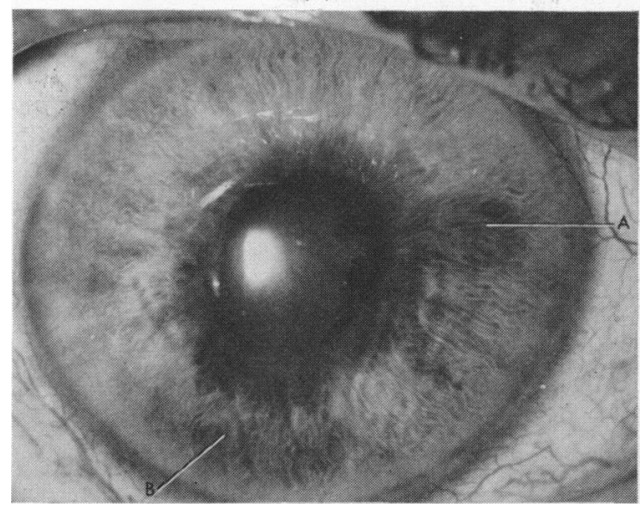

(c)

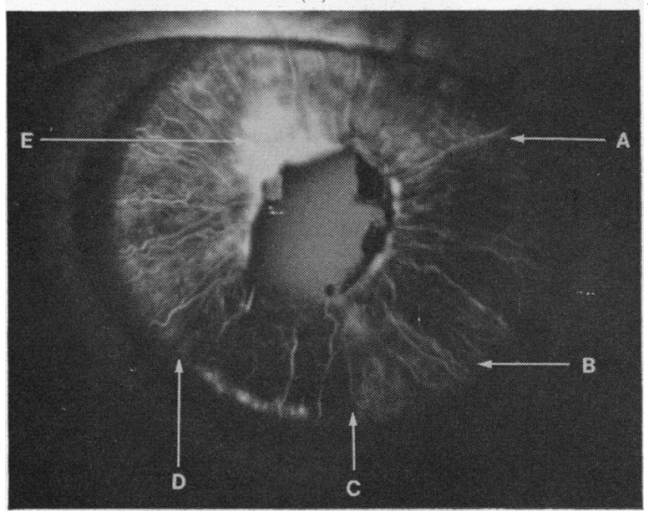

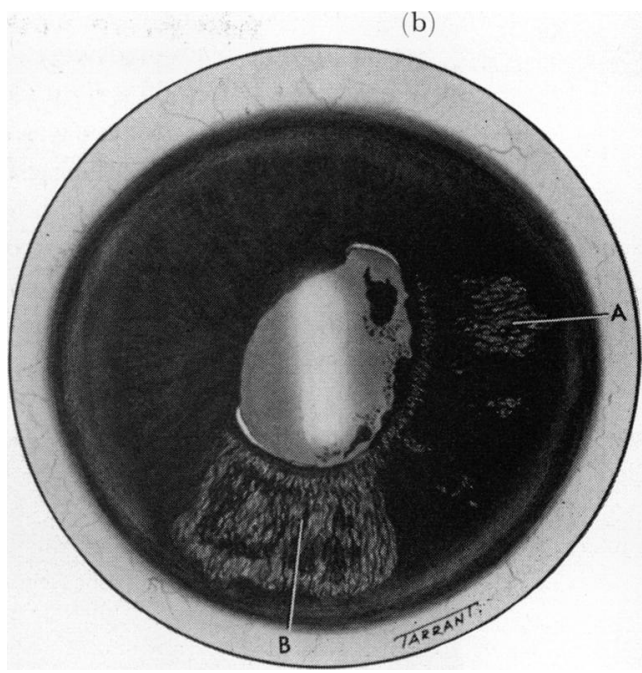

FIG. $4 a$ Right iris 6 months after an attack of anterior segment necrosis resulting from detachment surgery. $A$ and $B$ are sectors of atrophy affecting the iris stroma

FIG. $4^{b}$ Painting of transilluminated iris showing sectoral atrophy of pigmentary epithelium ( $A$ and $B)$

FIG. $4 c$ Angiogram showing sectors of ischaemia $(A-B ; C-D)$ and vascular leakage at pupil margin (E) (40 sec.)

A 57 -year-old male was treated by encerclage with a $3-\mathrm{mm}$. silicone strap in 1967 for a left retinal detachment, and the procedure was repeated for a recurrence of the detachment in $197 \mathrm{I}$.

After the second operation, the eye became hypotonic and developed heavy flare and cells in the anterior chamber, the patient being treated as a case of bacterial endophthalmitis. At a recent follow-up examination, the only significant abnormalities of the iris to be noted were sluggishness in the light response on the temporal side of the pupil with a small degree of atrophy of the pigmentary epithelium in the immediately adjacent iris. However, fluorescein angiography revealed a wide sector of incomplete filling (Fig. 5). It is therefore possible that the original postoperative condition was one of ishaemia rather than infection.

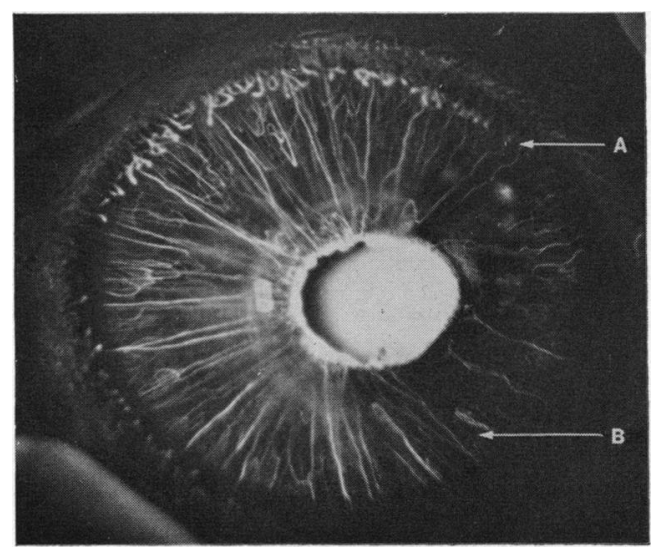

FIG. 5 Sectoral ischaemia $(A-B)$ in a patient 12 months after detachment surgery (36 sec.) 
Severe ischaemic changes were found in a patient who suffered a right traumatic carotico-cavernous $\frac{\overrightarrow{2}}{7}$ fistula in 1964 . In 1968 a right carotid ligation and muscle trapping procedure was performed, and $\underline{\underline{O}}$ in 1970 the left (opposite) eye was operated on for a left 6 th nerve (abducens) paralysis. The surgery involved removing and replacing the superior, inferior, and lateral rectus muscles. Postoperatively, $\stackrel{\vec{P}}{\longrightarrow}$ the classical signs of anterior segment ischaemia presented (Fig. $6 a$ ) with consequent sectoral atrophy믈 of the pigment epithelium and stroma of the iris (Figs $6 b$ and $c$ ). The iris angiogram (Fig. $6 d$ ) shows $\frac{\bar{\sigma}}{\omega}$. areas of leakage of fluorescein dye rather than ischaemia. This results from the masking of the $\frac{\widehat{\sigma}}{\widehat{\sigma}}$ intravascular fluorescence by the heavily pigmented stroma, so that individually fluorescing vessels $\cong$ cannot be seen.

(a)
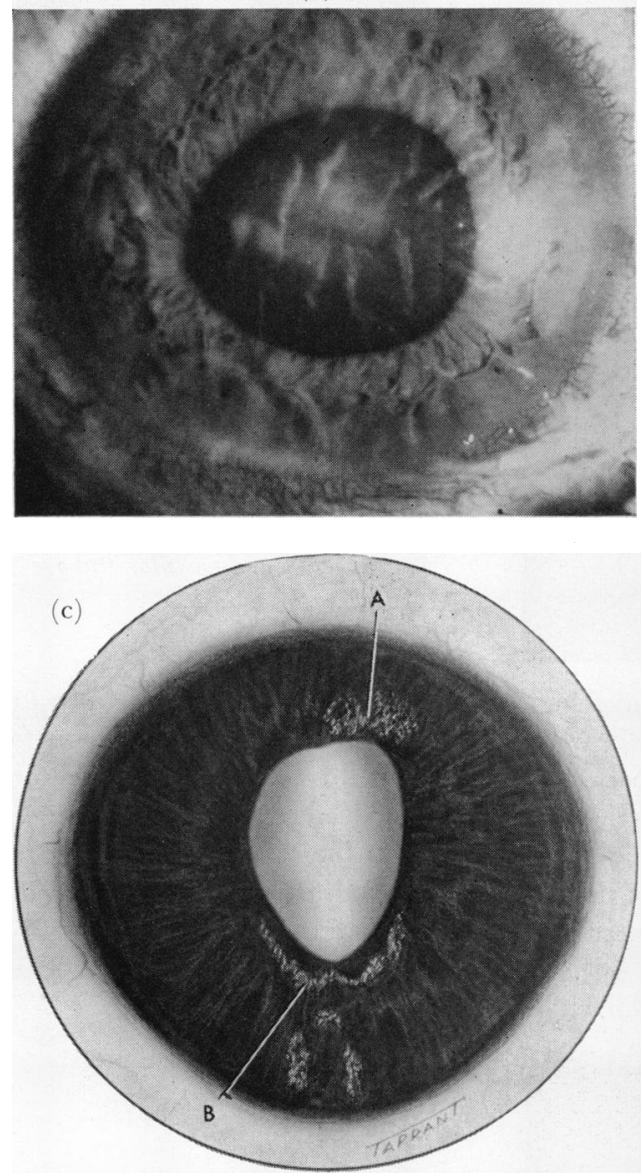

(b)

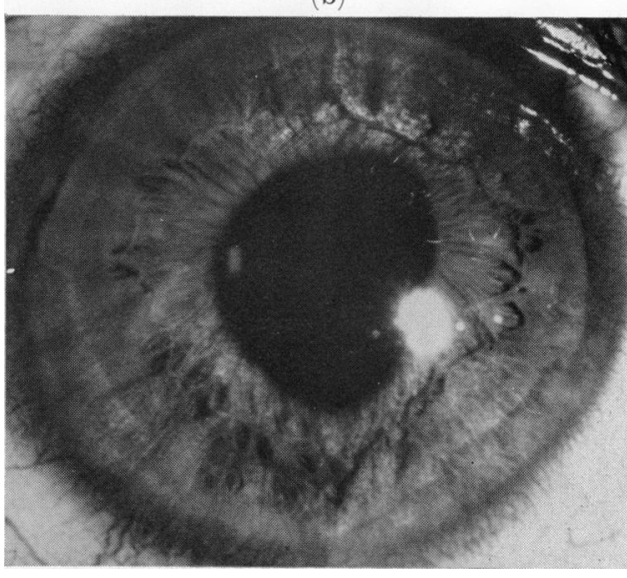

(d)

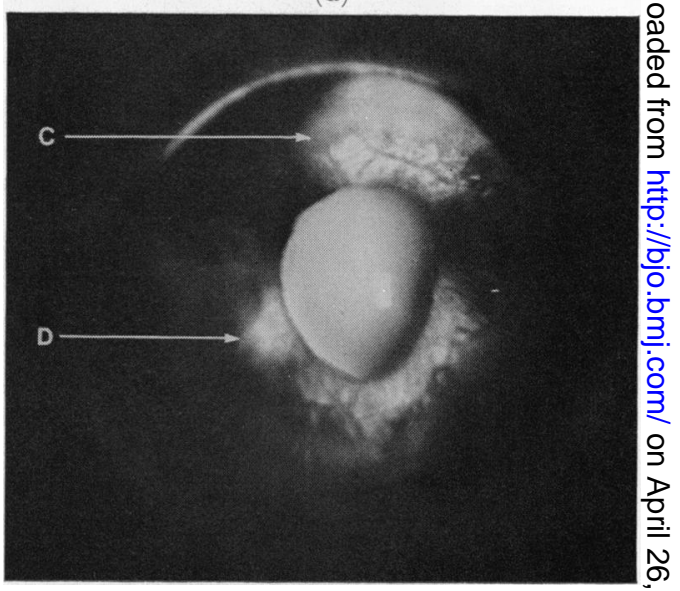

FIG. $6 a$ Ocular ischaemic syndrome after extraocular muscle surgery in the left eye of a patient treated for a N traumatic carotico-cavernous fistula on the opposite side

FIG. $6 b$ Clinical appearance 5 months later

FIG. 6c Painting of transilluminated iris, showing atrophy of pigmentary epithelium ( $A$ and $B$ )

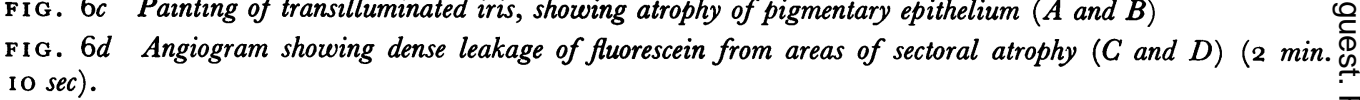

Fluorescein angiography as an aid to diagnosis

The preliminary investigation having shown that fluorescein angiography demonstrates ischaemia $\stackrel{\mathbb{\Phi}}{\mathbb{D}}$ in the diseased iris, its value was investigated as a diagnostic aid in patients in whom the diagnosis was not directly apparent. 
Fluorescein angiography was performed in a $5 \mathrm{I}$-year-old female with a clinical diagnosis of acute bacterial endophthalmitis after retinal detachment surgery. She was first treated unsuccessfully by external plombage in the upper temporal quadrant of the right eye; an encirclement was later performed with a $3-\mathrm{mm}$. silicone strap, and 3 days later the eye became acutely painful. The cornea became heavily infiltrated with inflammatory cells on the temporal side, while in the anterior chamber massive fibrinous exudation appeared. At this stage, because of the reaction in the cornea and anterior chamber, it was difficult to see the iris and to reach a diagnosis and the patient was treated with antibacterial therapy. Fluorescein angiography 4 days postoperatively demonstrated gross ischaemia. Fig. 7 shows the angiographic appearance ro days postoperatively when almost complete ischaemia of two-thirds of the total area is still in evidence. Thus angiography revealed the true nature of the acute postoperative inflammatory reaction.

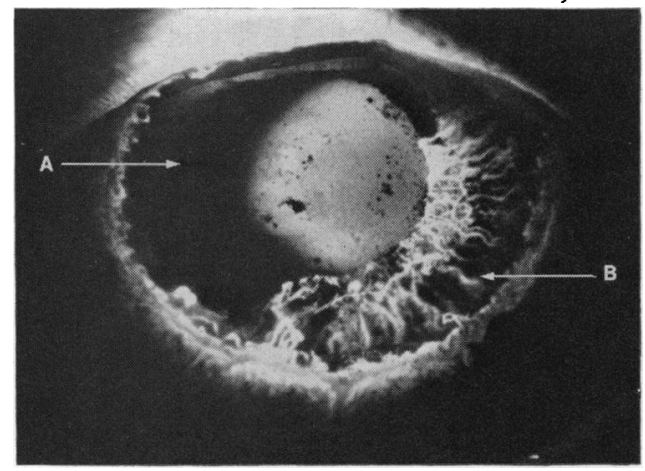

FIG. 7 Extensive ischaemia $(\boldsymbol{A})$ and vasodilatation $(B)$ in a patient with acute anterior segment necrosis after retinal detachment surgery (20 sec.)

In a similar situation, a $5^{1-y e a r-o l d ~ m a l e ~ w a s ~ t r e a t e d ~ w i t h ~ t w o ~} 5$-mm. radial plombs placed beneath the right lateral rectus muscle. Postoperatively the stromal oedema and folds in Descemet's membrane developed with a flare and cells in the anterior chamber. In the iris, there was bunching of the fibres of the stroma at 12 and 6 o'clock, with early atrophic changes in the pigmentary epithelium on the temporal side. An angiogram at the time showed definite ischaemic changes; it was of poor quality because of pain and photophobia and has therefore not been reproduced. Fig. 8 shows the angiogram 3 months after the attack, when the ischaemic area was essentially of the same distribution as in the postoperative angiogram. In this patient, a suspected diagnosis was confirmed and appropriate treatment was instituted.

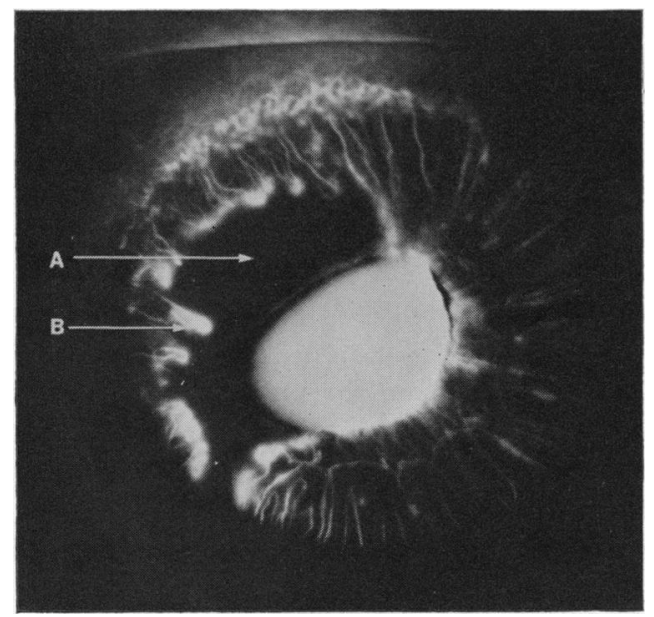

FI G. 8 Iris angiogram in a patient who had suffered acute ischaemia of the anterior segment 3 mths previously after retinal detachment surgery (25 sec.). (A) Area of ischaemia. (B) Marked leakage of fluorescein at vascular apices 
We are at the present time performing routine fluorescein angiography in patients with and without complications after retinal detachment surgery and comparing theo findings with the preoperative angiographic picture. Among the early changes that have so far been found in the absence of clinical signs is delayed filling in a sector of iris which corresponds to the meridians between which an external plomb was applied.

Fig. 9 demonstrates such an area of late filling in the supero-temporal quadrant of the left eye in a⿸户 20-year-old male who had been treated by external plombage for a retinal dialysis; I 5 sec. later the vessels of this quadrant had become well perfused with dye.

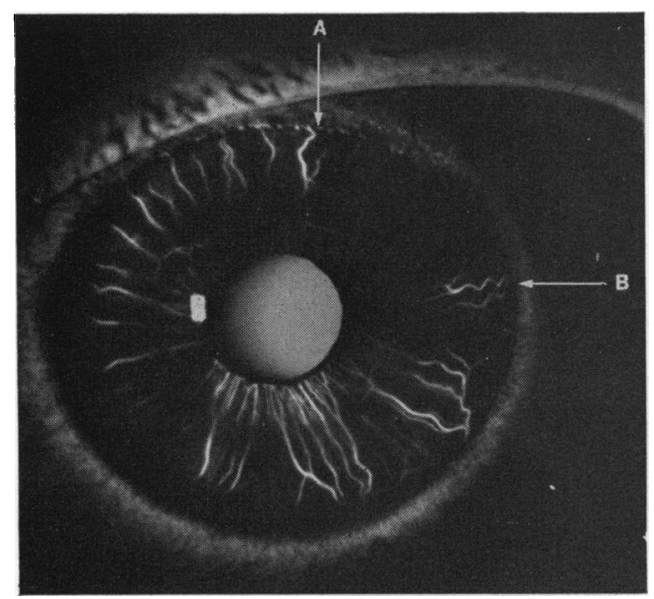

FIG. 9 Iris angiogram demonstrating area of transient ischaemia $(A-B)$ in relation to an ex- ternal plomb placed in the same sector

\section{Discussion}

The present study has shown that fluorescein angiography may be used as a method oß demonstrating ischaemic areas in the iris. When the sector of iris which is affected is small, only the pigmentary epithelium is affected and the area of atrophy may coincide closely with the avascularity as shown by angiography. As the extent of vascular involve $\frac{0}{0}$. ment becomes greater, the sphincter pupillae becomes clinically affected; at the lowes level this presents as a slight defect in the pupil in response to a light stimulus in the ischaemic sector, but in severe cases the classical clinical appearance producing an ova年 semi-dilated pupil with distortion and bunching of radial stromal fibres superveneso Visible changes occur in the iris stroma only after the more severe attacks. In a pig mented iris the superficial layer of stromal pigment becomes aggregated into granular clumps and exposes pale unpigmented fibres of the iris stroma beneath, but, in a blus iris, ischaemia may merely cause a thinning of the stromal fibres.

During an attack of ischaemia, the vessels in the unaffected portion of the iris show intense intravascular fluorescence which suggests some degree of vasodilatation. Extensive leakage can also occur from the patent vessels in non-ischaemic sectors, suggesting change in their permeability that might result from the production of pharmacologicallye active substances in the ischaemic areas. Once the acute inflammation has died down, the vasodilatation disappears, even though extensive areas of ischaemia persist as a permanen?्ष change. Areas of leakage may also remain, although these are less severe. It is note $\frac{\mathcal{P}}{\mathbb{D}}$ worthy that many of the patients who have been studied have shown little evidence of neovascularization resulting from an ischaemic stimulus. 
The clinical presentation of anterior segment necrosis is often not clear-cut, and it is in the early stages of this complication that urgent treatment is required. Fluorescein angiography can be performed as a diagnostic procedure using any suitably adapted slit-lamp microscope and does not necessarily require to be used in conjunction with sophisticated photographic recording equipment. Thus, with appropriate barrier and excitor filters fitted on to the microscope eye-pieces and light source, a clinician can easily observe the vascular characteristics of the iris vessels after an intravenous injection of fluorescein. Such an examination could have a critical influence on the future progress of the patient, and the eventual visual result.

The study of fluorescein angiography of the iris is of particular value after operations for retinal detachment, in which it is often difficult to ascertain the cause of acute inflammation of the anterior segment.

The cause of anterior segment necrosis after retinal detachment surgery is difficult to explain by obstruction of the long posterior vessels alone, when it is remembered that the iris receives a proportion of its blood supply from the anterior ciliary vessels and that the iris has a good anastomosis in its greater and lesser arterial circles. Sanders and Hoyt ( I969) have lucidly discussed the vascular mechanics involved in maintaining adequate circulation of blood in the normal eye and in patients with carotico-cavernous fistulae who have undergone surgery to occlude the fistula. They discuss the relationship of the pulse pressure and peripheral resistance to the circulation of blood, and point out that a reduction in the pulse pressure caused either by a fall in the arterial pressure or by a rise in the venous pressure, if not compensated for by a decrease in peripheral resistance, will produce ischaemia. The influence of the intraocular pressure is critical, in that its elevation is associated with a commensurate rise in the venous pressure of the intraocular vessels. Hence, in a situation where the pulse is reduced, a rise in the intraocular pressure, though small, may precipitate an attack of ischaemia. The pulse pressure in the vessels feeding the anterior segment with blood is likely to be reduced after retinal detachment surgery and a small transient rise in intraocular pressure may easily tip the balance in favour of a manifest ischaemic episode.

In cases of ischaemia after retinal detachment operations, obstruction of the long posterior ciliary arteries and possibly of the vortex veins may occur, but to account for the changes found in the anterior segment, and in particular, in the iris, some obstruction of the anterior ciliary circulation may be an additional factor.

Thus it is possible that undue handling of the extraocular muscles during surgery may alter the vascular dynamics and cause temporary obstruction of blood flow in the vessels. Changes in the anterior ciliary vessels as well as in the long posterior ciliary vessels would then produce the clinical appearances that have been described. Such changes in the anterior ciliary vessels need be only temporary, and if accompanied by a transient rise in intraocular pressure, may play a part in the pathogenesis of anterior segment necrosis. The avoidance of unnecessary manipulation or removal of extraocular muscles during retinal surgery is therefore stressed.

\section{Summary}

The vascular characteristics of the iris in patients with ischaemia of the anterior segment have been investigated by means of fluorescein angiography. Distinct areas of ischaemia can be demonstrated, and it is pointed out that the method is a useful diagnostic aid in patients with inflammatory signs after retinal detachment surgery, some of whom may 
have anterior segment necrosis. The mechanism of this complication of detachmene surgery is discussed and it is suggested that, in addition to long posterior ciliary arteryo obstruction, undue handling of the extraocular muscles during surgery may play a part in its pathogenesis. Detachment of the extraocular muscles from the globe should clearly be avoided.

The help and encouragement of Prof. Barrie R. Jones and Mr. L. G. Fison is gratefully acknowledged. W are indebted to the Audio-Visual Department of the Institute of Ophthalmology for help in preparing the illustrations, and to Mr. T. Tarrant who illustrated the transilluminated iris. We are gratefully indebted ten The Clothworkers' Company for the partial financial support of this project.

\section{References}

BAGgesen, L. H. (1969) Acta ophthal. (Kbh.), 47, 449

boniuk, M., and zimmerman, L. E. (196i) Arch. Ophthal. (Chicago), 66, 318

BRON, A. J., and EASTY, D. L. (1970) Trans. ophthal. Soc. U.K., 90, 339

GHENG, H., BRON, A. J., and EASTY, D. L. (197I) Ibid., 91, I99

CHIGNELL, A. H., and EASTY, D. L. (1971) Ibid., 91, 243

COBв, в. (1968) Ibid., 88, 2 I I

- , shilling, J. s., and chisholm, I. H. (1970) Amer. F. Ophthal., 69, 573

CROCK, G. (1967) Trans. ophthal. Soc. U.K., 87, $5^{13}$

DÈODATI, F., BEC, P., LABRo, J.-B., and CAMEZIND, K. (1969) Bull. Soc. Ophtal. Fr., p. Iog9

JENSEN, v. A., and LUNDBAEK, K. (1968) Diabetologia, 4, I6 I

KNOX, D. L. (1965) Amer. F. Ophthal., 60, 995

mitsui, y., matsubara, m., and kanagawa, m. (1969) Brit. F. Ophthal., 53, 505

o'DAY, D., HOYT, W. F., and GRock, G. (1970) Med. F. Aust., 2, 460

SANDERS, M. D., and hоYт, w. F. (1969) Brit. F. Ophthal., 53, 82

vanNas, A. (1969) Acta ophthal. (Kbh.), Suppl. ro5, p. 24

wilson, w. A., and IRvine, s. R. (1955) Trans. Amer. Acad. Ophthal. Otolaryng., 59, 50 I 